\title{
Participación política y luchas por el sufragio femenino en Argentina (1900-1947) $)^{1}$
}

\section{Dora Barrancos ${ }^{2}$}

Recepción: 17 de septiembre de 2013 / Aprobación: 10 de enero de 2014

\section{Resumen}

Este artículo analiza la historia de la agencia feminista por los derechos políticos en la Argentina hasta 1947. En los primeros años del siglo XX se constituyeron los primeros grupos feministas. Muchas mujeres se adhirieron al socialismo y al librepensamiento, y aunque todas estaban de acuerdo con los derechos civiles, habían tenido algunas discrepancias con respecto a apoyar el voto. Alicia Moreau, quien se convirtió en la más importante de las socialistas feministas, creía que el voto debía llegar por etapas. Pero después de la Primera Guerra Mundial, todas las feministas argentinas abogaban por el derecho universal del sufragio en igualdad con los varones. La década de 1920 fue muy significativa en la movilización por ese derecho y aunque la Cámara Baja sancionó el sufragio en 1932, el Senado no trató la cuestión.

Fue el gobierno de Juan Domingo Perón en 1947, el que sancionó el sufragio femenino. Se destaca la notable figura de Eva Perón que creó la Rama Femenina del Partido Justicialista. En 1951 cuando las mujeres votaron por primera vez en la Argentina, ocuparon más del 30\% de los escaños parlamentarios. Se trató de un acontecimiento singular, pues ningún país de América Latina poseía, a mediados del siglo pasado, esa cantidad de representantes mujeres.

\section{Palabras clave}

Feminismo, feministas argentinas, derechos de las mujeres, sufragio, peronismo, Siglo XX

\section{Abstract}

This article analyzes the history of feminist agency for political rights in Argentina until 1947. In the early years of the twentieth century, the first feminist groups were created. Many women adhered to socialism and free thought, and although they all agreed with civil rights, they had some disagreements about supporting the vote. Alicia

$1 \quad$ Una primera versión de este trabajo fue presentado como conferencia en el "Encuentro Mujeres a las Urnas: 60 aniversario de elegir y ser electas en Costa Rica", con el auspicio del CIICLA, Universidad de Costa Rica, 27-29 de agosto del 2013.

2 Argentina. Socióloga y Doctora en Historia por la Universidade Estadual de Campinas, Brasil. Profesora Consulta de la Facultad de Ciencias Sociales de la Universidad de Buenos Aires, Argentina. Investigadora Principal y Directora en representación de las Ciencias Sociales y las Humanidades desde mayo del 2010 en el Consejo Nacional de Investigaciones Científicas y Técnicas. Correo electrónico: dora1508@aol.com 
Moreau, who became the most important of the socialist feminists, believed that the vote should come in stages. But after World War I, all argentine feminists advocated the universal right of suffrage in equality with men. The mobilization in the 1920s was very significant for that right and although the Low Camera passed the vote in 1932, it did not address the issue to Senates.

It was the government of Juan Domingo Perón in 1947, which sanctioned women's suffrage. It highlights the remarkable figure of Eva Peron who created the Rama Femenina del Partido Justicialista. In 1951 when women voted for the first time in Argentina, occupied more than $30 \%$ of the parliamentarian seats. It was a singular event, since no country in Latin America had, at mid-century, that number of women representatives.

\section{Keywords}

Feminism, argentine feminists, women's rights, suffrage, peronism, 20th century

\section{Resumo}

Este artigo analisa a história da agência feminista dos direitos políticos na Argentina até 1947. Nos primeiros anos do século XX emergiram os primeiros grupos feministas. Muitas mulheres aderiram ao socialismo e ao livre pensamento e, ainda que todas estivessem de acordo com os direitos civis, tiveram algumas divergências com relação ao apoio ao voto. Alicia Moreau, que se tornou a mais importante das feministas socialistas, acreditava que o voto deveria chegar em etapas. Mas após a Primeira Guerra Mundial, todas as feministas argentinas passaram a defender o direito universal do sufrágio em igualdade com os homens. A década de 1920 foi muito importante na mobilização por esse direito, e apesar da Câmara dos Deputados ter aprovado o sufrágio em 1932, o Senado não tratou o tema.

Foi o governo de Juan Domingo Perón, em 1947, que sancionou o sufrágio feminino. Destaca-se a notável figura de Eva Peron que criou a Rama Feminina do Partido Justicialista. Em 1951, quando as mulheres votaram pela primeira vez na Argentina, ocuparam mais de $30 \%$ dos assentos parlamentares. Foi um fato singular, uma vez que nenhum país da América Latina possuía, em meados do século passado, essa quantidade de representantes mulheres.

\section{Palavras chave}

Feminismo, feministas argentinas, direitos das mulheres, sufrágio, peronismo, século XX

\section{Los primeros pasos del feminismo en la Argentina}

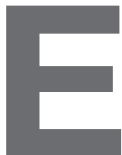

ntre las notas de la Argentina "moderna" se encuentra la recepción del feminismo, término que -tal como he descrito en otro lugar (Barrancos, 2005b)- fue de inicio polisémico y en verdad poco ajustado al significado que hoy le otorgamos. Hacia fines del XIX algunas mujeres de la élite, seguramente conocedoras del uso de la noción en la 
sociedad francesa -a la que solían visitar asiduamente- la incorporaron de modo "naif" para connotar ciertas experiencias completamente estereotipadas del género. En esos círculos se asimilaba "labores femeniles" a "feminismo". Pero fueron las socialistas y las librepensadoras -y sobre todo las más letradaslas primeras adherentes de las ideas y propósitos que se disponían a enfrentar, la desigualdad ominosa que padecían las mujeres y el sometimiento al orden patriarcal. Podría asegurar que la incorporación al feminismo significó adherirse a una forma de actuación política y que en todo caso hay una larga tradición de participación política de las mujeres en la Argentina, en todos los tiempos y desde mucho antes que hubieran conquistado derechos y ciudadanía.

El Partido Socialista, creado en 1896, fue el primero en proponer el voto de las mujeres en la Argentina, tal como lo habían hecho los partidos homólogos europeos, y no debe entonces extrañar que sus adherentes mujeres se tornaran feministas. A menudo se cruzaban la denominación de "socialista" y "feminista", y no pocas veces con propósito estigmatizante, como cabe imaginar. Muchas librepensadoras, por fuera del socialismo -entre quienes deben contarse las que se incorporaron a la "masonería por adopción"- se convirtieron también en feministas. Pero debe reconocerse que hay por lo menos dos feministas de la primera hora que difícilmente puedan ser encuadradas como socialistas o librepensadoras, me refiero a Cecilia Grierson - la primera médica formada en la Argentina- y Elvira Rawson de Dellepiane, también médica quien estuvo inicialmente vinculada a la naciente fuerza política, la Unión Cívica Radical. Cecilia fue una de las creadoras del Consejo de Mujeres a inicios del siglo XX, pero luego se apartó porque el organismo quedó en manos de mujeres más conservadoras.

Rescataré algunos nombres fundamentales de la saga de la primera hora en materia de derechos de las mujeres haciendo foco en el problema del sufragio, con el ánimo de mostrar que no siempre hubo concordancia acerca de las circunstancias del voto de las mujeres en el propio seno del movimiento feminista argentino. No pueden disimularse las discrepancias iniciales sobre la oportunidad y alcance de la ciudadanía, aunque, y tal como desarrollaré, la Primera Guerra Mundial y las notables transformaciones mundiales y locales ocurridas a fines de la década de 1910, significaron un nuevo cauce para la perspectiva común del sufragio.

María Abella Ramírez, uruguaya de nacimiento, vivió largos años en La Plata, una ciudad inaugurada a fines del siglo XIX, de acendrado carácter universitario y donde se deseaba consagrar el espíritu de la modernidad laica (Vallejos, 2007, 7-17), la cual se consagró por completo a la agencia por la igualación de los sexos. María editó en 1901 la primera publicación periódica que se declaró "feminista", la revista Nosotras cuyo objetivo fue servir de impulso a la igualación civil y política de las mujeres. Tampoco faltaron en sus páginas alegatos contra la violencia que estas padecían, denunciando las prerrogativas penales de que gozaban los maridos victimarios. Otro aspecto de la publicación 
se refiere a los debates que promovía, entre los que se destaca uno muy prolongado dedicado a la vinculación religiosa.

María estuvo entre las primeras feministas que solicitaron, además de la reforma del Código Civil -sancionado en 1869 y que, al igual que el Código francés, determinaba la inferioridad jurídica a las mujeres-, el sufragio universal femenino, esto es sin ningún tipo de limitaciones censitarias. Años más tarde, en 1909, en otra publicación que dio continuidad a Nosotras, y a la que puso el nombre de La Nueva mujer (una síntesis de su apuesta a las transformaciones que veía en las congéneres), sus posiciones fueron más incisivas en materia de voto femenino. Debe recordarse que en esos años iniciales del siglo XX, la Argentina acogió a la española Belén de Sárraga, una de las figuras internacionales más destacadas en la lucha por el sufragio femenino. Belén visitó la enorme mayoría de nuestros países -tal vez estuvo presente en todos-, ejercitando su verbo encendido, de bella articulación, en el que alegó a favor de los derechos cívicos de las mujeres. Sabemos que su encuentro con las argentinas fue un estímulo decisivo en la petición del voto.

Entre las primeras sufragistas argentinas también se encuentra Julieta Lanteri, quien había emigrado desde Italia siendo pequeña y se formó como médica en la Universidad de Buenos Aires. Julieta muy joven inició el camino para arribar a la ciudadanía. En 1911 pudo, de modo excepcional, emitir su voto en las elecciones de representantes de la ciudad de Buenos Aires -en esa ocasión se permitió votar a los extranjeros y como tal pudo participar-. Su empeño a favor del sufragio la llevó a pleitear judicialmente la cuestión, y en un célebre fallo el juez adujo que la Constitución no hacía distinción de sexos, pero que dado que era inescindible del atributo de la ciudadanía la conscripción militar obligatoria -era ciudadano quien podía ser movilizado militarmentetal la interpretación sobre la obligación del servicio militar, allí residía la inhibición para las mujeres. Julieta se presentó entonces a un cuartel solicitando ser admitida para cumplir con la exigencia legal, pero no llegó siquiera a ser escuchada por la jefatura del organismo (Bellota, 2000, 143-150).

A la lucha por los derechos de las mujeres también se unió muy joven Alicia Moreau, quien sería reconocida como la más destacada voz femenina del socialismo en la Argentina. Hacia 1909 ella conducía, junto con Enrique del Valle Iberlucea -una figura notable entre otras cosas por su alianza con las mujeres- la revista Humanidad Nueva, sucedánea de otra célebre publicación, la Revista Socialista Internacional. En aquel período, Alicia estaba convencida de que el mayor auxilio que debía prodigarse a las mujeres era la educación. La gran gesta era contribuir a esclarecerlas, hacerlas partícipes del conocimiento, puesto que -solía asegurar a la manera iluminista del período- era la vía más directa y más sólida hacia la emancipación. Alicia apoyaba el sufragio pero creía, como muchas feministas del momento, que la conquista debía hacerse por etapas a fin de que las mujeres tuvieran tiempo de aprender acerca de la arena política. Además, Alicia aseguraba que lo mejor era iniciar la experiencia 
de voto en el orden comunal, de modo que luego se accediera al orden provincial, hasta mucho más adelante llegar a ejercitarlo en el ámbito nacional (Barrancos, 2005a, 167-168).

En 1910 tuvo lugar el Primer Congreso Femenino en Buenos Aires con la presencia de mujeres de diversos países, especialmente de América Latina, o al menos a través del envío de ponencias para quienes no pudieron allegarse. Fue una experiencia singular propuesta por la Asociación de Universitarias Argentinas, que se desarrolló a través de una serie de secciones donde era necesaria la inscripción de las ponencias.

Por lo tanto, con respecto a la cuestión del sufragio femenino en la Argentina, no hubo unanimidad en todos los segmentos, y no seríamos fieles a los mejores trazos de la tradición feminista si no mencionáramos las dificultades para ponerse de acuerdo sobre esta demanda. Es necesario subrayar el papel jugado en el Congreso de 1910 por la Liga Nacional de las Mujeres Librepensadoras, entidad que demandó de modo irrestricto el derecho al sufragio a través de las representantes María Josefa González y Ana de Montalvo.

Del mismo modo, destaca la posición del Centro Socialista Femenino -uno de los más importantes nucleamientos de mujeres del Partido-, por entonces y sin duda uno de los estimuladores del Congreso, cuya solicitud a favor del voto se hizo especialmente a través de Raquel Messina -una militante de particular enjundia intelectual-. El Centro había sido taxativo en su solicitud, y el derecho al sufragio universal igual para varones y mujeres y aparecía en el primer lugar de sus demandas. Estas manifestaciones pudieron confrontar con las de la propia Alicia Moreau, y no apenas con ella. En el orden de prioridades del feminismo del Centenario, la protección de las madres y de los niños desvalidos, la elevación educativa y desde luego, la modificación del Código Civil, parecían encabezar la agenda. De esta manera, sin duda, el debate sobre la oportunidad y alcances del sufragio, dividía las aguas de nuestras primeras militantes por las causas de las mujeres.

\section{La madurez del feminismo en la década de 1920}

Al finalizar la Gran Guerra se asistió a los bien conocidos cambios sufridos por las sociedades occidentales. Inglaterra, en donde los combates sufragistas habían sido tan largos, intensos y conmovedores, se sancionó finalmente el voto aunque con limitaciones. La orientación de las organizaciones feministas más importantes de la Argentina se tornó unánime respecto del voto universal de las mujeres. Se ha evidenciado, que la década de 1920 dejó traslucir transformaciones de gran significado en la lucha por la conquista de derechos (Lavrin, 2005; Barrancos, 2007). En 1919 se presentó el primer proyecto de sufragio y en el transcurso de la década de 1920 se sumaron otras iniciativas. Fue un período en el que la agitación por las prerrogativas de las mujeres aumentó considerablemente, aunque subrayada en Buenos Aires, en 
donde hubo escenas ficcionales de voto llevadas a cabo por las organizaciones a cuyo frente estaban Elvira Rawson de Dellepiane, Julieta Lanteri y Alicia Moreau. Esta última se casó a inicios de esa década con el más destacado socialista -cabeza del partido- el doctor Juan B. Justo.

La época había transformado en algún grado las costumbres sexuales rígidas, y aunque se seguía exigiendo una moral impoluta a las mujeres, no pocas pudieron transgredir las convenciones por lo que se renovaban las voces condenatorias, en particular del feminismo al que hacían responsable de los descalabros que creían ver en mujeres más independientes. La moda favoreció las faldas y cabelleras cortas -un símbolo de la antigua configuración de "lo femenino" (Perrot, 2008)- pero también se seccionaron. Los nuevos medios, especialmente el cine, permitieron la difusión de escenas con actrices osadas, que permitían al menos ensoñaciones de cambios de subjetividad, y hasta de vida. Surgió, gracias a Julieta Lanteri, el Partido Feminista y aunque tuvo oposiciones de algunas feministas y de ciertos segmentos progresistas - las fracciones de la masonería que defendían el sufragio no comulgaron con la idea de un partido- la nueva fuerza surgía con ímpetu y se asimilaba a otras experiencias internacionales.

En conjunto, nuestras feministas avanzaron en el transcurso de esos años y pudieron reunir nuevos grupos de mujeres provenientes de los sectores medios más elevados. Hasta la Iglesia había cambiado bastante de opinión y algunos obispos autorizaban la solicitud del sufragio, seguramente animados por las voces de no pocos progresistas que azuzaban con el fantasma de que el voto de las mujeres era lo más seguro para consagrar las ideas conservadoras. Lo cierto fue que las fuerzas de derecha, ocasionaron el primer golpe militar de la Argentina en 1930, con el derrocamiento del gobierno radical de Hipólito Yrigoyen -quien en verdad no estaba a favor del voto de las mujeres y tampoco del divorcio- circunstancia que exhibe las dificultades de orientación de las fuerzas políticas liberales en mi país.

Es necesario introducir, que en la provincia de San Juan se otorgó el voto a las mujeres en 1928 en circunstancias muy particulares. La deposición del gobierno radical en aquella provincia originó un movimiento a cuyo frente estuvieron los hermanos Federico y Aldo Cantoni -este último había militado en el socialismo- y que seguramente por razones especulativas otorgaron el voto femenino. En 1934, la abogada Emar Acosta accedió por primera vez a una banca representacional, sin embargo, el voto se ejercitó y se retrajo de acuerdo con las sucesivas intervenciones que sufrió la provincia.

En el ámbito nacional, en 1932, y a pesar del avance de las derechas, hubo ocasión para que la Cámara Baja tratara el sufragio femenino y el divorcio vincular. En materia de divorcio la Argentina estaba claramente atrasada en relación con Uruguay, que lo había consagrado en 1907 y ampliado sucesivamente 20 hasta 1912, año en que se sancionó la norma que posibilitaba la obtención 
del divorcio a sola petición de la mujer. Frente al debate en el Parlamento, la movilización femenina se intensificó, aunque las organizaciones de mujeres más conservadoras abjuraran del divorcio y apoyaran el sufragio desde que se subiera la edad para las mujeres. Finalmente, hubo media sanción para esas iniciativas, pero no se abrió el debate en el Senado en donde eran absoluta mayoría las fuerzas más conservadoras (Palermo, 1998).

\section{Perplejidades del feminismo y conquista del sufragio bajo el signo del peronismo}

He sostenido que después del fracaso y durante la década 1930 e inicios de los años 40, las feministas argentinas mitigaron bastante los reclamos por el sufragio, toda vez que sus esfuerzos estuvieron dedicados especialmente a la lucha antifascista y, de modo particular, a apoyar a las fuerzas republicanas cuando se desató la Guerra Civil Española (Barrancos, 2007, 174-175). La sintonía de nuestras feministas con el auxilio a sus víctimas fue singular, dando lugar a diversas organizaciones entre las que alcanza preeminencia la Junta de la Victoria (Mc Gee Deutsch, 2007). En efecto, se trató de un extenso movimiento de mujeres que se extendió a todo el país, aunque con foco en las grandes ciudades, especialmente en Buenos Aires. El agrupamiento convocó a un variopinto conjunto de socialistas, liberales, incluyendo a quienes tenían inscripción confesional, y desde luego no faltaron las católicas que repudiaban los regímenes autoritarios de derecha.

Si se vuelve sobre las páginas de Vida Femenina, la principal publicación de las socialistas (1933-1943), a cuyo frente estaba María Luisa Berrondo, se podrá constatar que el eje de las preocupaciones se ha dislocado para la denuncia de los regímenes reaccionarios, y las prevenciones acerca de la proliferación de sus tentáculos en el propio país; y el nuevo golpe de Estado de 1943 pareció la profecía cumplida. Hubo un interregno de incubamiento del fenómeno que sería conocido como "peronismo", a propósito del posicionamiento paulatino del coronel Juan Domingo Perón, quien llegó a ocupar la Secretaría de Trabajo y Previsión, consiguiendo una articulación directa con las fuerzas trabajadoras en plena expansión.

Debe recordarse que las primeras organizaciones obreras se constituyeron en los inicios del siglo XX, merced a la inmigración ultramarina masiva que caracterizó a la Argentina por lo menos hasta el inicio de la Primera Guerra Mundial. Se trató de una población que modificó sustancialmente el escenario social de la Argentina, sobre todo en los grandes centros urbanos. Anarquistas, socialistas y sindicalistas revolucionarios habían sido las principales orientaciones de las fuerzas del trabajo, pero el inicio del ciclo de la "sustitución de importaciones", que se dilató desde mediados de la década de 1930, modificó los perfiles de las y los obreros y el orden de las reivindicaciones. 
En octubre de 1945 se produjo la detención del ya bien conocido coronel Perón debido a la desconfianza que inspiraba en las propias fuerzas armadas. De allí en adelante, la historia se precipitó cuando tuvo lugar la notable movilización popular, que culminó el 17 de octubre con su liberación y la construcción de la fuerza que lo catapultó como presidente en 1946. Perón había conocido a la actriz María Eva Duarte de modo azaroso, en un acto de ayuda a los damnificados por el terrible terremoto que asoló justamente a San Juan en 1944, y más tarde se casó con ella.

Casi todas las fuerzas políticas que disputaron la contienda electoral de 1946 habían prometido el voto a las mujeres. El triunfo de Perón, y el acicate de su esposa que en pocos años se convertiría en el ariete más estratégico del régimen, aseguraron el camino hacia el sufragio. Desde luego, contaba el Acta de Chapultepec de 1945, el compromiso de los países de la región de saldar la deuda con la ciudadanía de las mujeres, y la iniciativa llegó al Congreso Nacional en 1947. Eva había realizado un viaje a Europa, con ciertas proyecciones celebratorias a mediados de ese año, dado que Perón tenía planes de reacomodar la economía nacional en orden a las nacionalizaciones, la creación de empresas estatales, la profundización del proceso sustitutivo de importaciones para desarrollar la industria nativa, y se imponían pactos con los países que vivían la posguerra.

Eva había pedido especialmente que el debate comenzara a su regreso, pues quería acompañar de modo activo las diferentes fases del tratamiento, y tal vez asegurarse de que se sancionaría como había sido previsto. Eva visitó sindicatos y fábricas nutridas de mujeres para agitar a favor del sufragio, las movilizó a la Plaza de los dos Congresos en apoyo de la medida, las acompañó sentada en el palco de los debates hasta la sanción el 9 de septiembre, y unos días más tarde en aquella primavera, habló a una multitud desde el balcón de la Casa Rosada cuando su marido promulgó la Ley 13010.

Había, sin duda, mucha inquietud entre las feministas que recelaban completamente del régimen. Socialistas y liberales sostenían que la medida sería manipulada, y se trataba, tan luego, de la vieja aspiración sostenida desde inicios del siglo, como se ha mostrado. Lo cierto es que más allá de las prevenciones, la figura de Eva se proyectó notablemente en la escena nacional. Eva fue una figura sin duda excepcional, por su profunda intuición y la rapidez del aprendizaje de las interacciones del poder que la consagraron. Había vivido en carne propia las exclusiones, comenzando por su situación familiar -era hija natural de un pequeño hacendado de la provincia de Buenos Aires-. Su dislocamiento hacia la gran urbe, siendo muy joven, y la inscripción en el mundo de la actuación escénica, fueron particularmente difíciles en esos años, como ha mostrado la bibliografía que se le ha dedicado (Navarro, 1981; Pichel, 1993; Dujove Ortiz, 1995). El encuentro con Perón seguramente politizó sus fibras intuitivas, y se tornó un auxiliar fundamental en la concepción redistributiva del régimen haciendo más urgente la "justicia social". Inspirada en el 
modelo falangista de la "sección femenina", decidió la construcción de la Rama Femenina del Justicialismo mediante una serie de pasos exitosos, comenzando por la designación de las censistas que irían a todos los lugares del país a fundar la Rama (Barry, 2009, 109-111).

Las concepciones de género de Evita son, por cierto paradójicas y han sido objeto de diversas interpretaciones (Valobra, 2010). Si por una parte, hubo evidencias sobradas acerca del arraigo de las claves de los estereotipos, por otra, fueron prominentes los sacudones que efectuó. Piénsese en la manera como organizó a miles y miles de mujeres en todo el país, desarrollando la estrategia de las Unidades Básicas Femeninas -lugares a los que exigió que no tuvieran la presencia de varones para evitar las malidicencias- (Barry, 2009, 157-177), haciéndolas células de adhesión a Perón y su causa, y reclamando a las congéneres una completa adhesión que podía significar "dar la vida por Perón". Si el mandato era la construcción de "hogares regulares", la dedicación inalterable a la vida familiar y la entrega a los suyos de manera abnegada, tal mandato entraba en completa crisis frente a la exigencia de "abandonarlo todo", para contribuir con la propia vida al sostenimiento de la "Nueva Argentina de Perón". Evita misma era una prueba de ese oxímoron, entre lo recóndito privado y la máxima exposición pública que le significaron los peores ataques, las formas más terribles de vilipendio y condena. Los sectores opositores nunca dejaron de verla como la peor figura del régimen, y cuando se supo que estaba atacada por un cáncer-que acabaría con su vida en forma temprana-un grafitti incalificable celebró "Viva el cáncer". Tal era el carácter de la denostación implacable que se le ofrendaba.

En 1948, cuando se estaba en pleno proceso de confección del empadronamiento femenino, hubo que tratar en el Congreso una rectificación de la ley electoral que exigía la exhibición del año de nacimiento de los ciudadanos en los padrones, que en verdad, y debido a la asimilación con el ya introducido servicio militar obligatorio, se mostraba bajo el "número de clase". El debate alcanzó momentos fulgurantes desde la perspectiva de las relaciones de género, pues para muchos representantes era menester corregir la alevosía de indicar la fecha de nacimiento, teniendo en cuenta "la naturaleza sensible" de las mujeres sobre este particular ángulo. Y desde luego, por "cortesía al sexo débil", se sancionó la ley que eliminaba la información de la fecha de nacimiento en los padrones femeninos (Barrancos, 2002, 147-149).

El voto de las mujeres ocurrió por primera vez el 11 de noviembre de 1951 y contra lo que algunas voces preveían, reveló especial entusiasmo y muy elevada concurrencia; su resultado fue el fortalecimiento de la candidatura del general Perón. Pero la singularidad de ese primer desempeño estuvo dada por la asignación de cuotas internas dentro del Partido Justicialista, lo que permitió que más de 30\% de candidatas llegara a ocupar escaños en ambas Cámaras del Congreso Nacional. En efecto, el Justicialismo estaba compuesto por tres Ramas, la de los varones, la sindical y la femenina, y la astucia de Evita llevó al 
acuerdo de que debería haber igualdad en la distribución de las bancas, por lo que correspondió a las mujeres la cuota correspondiente. Aunque la Argentina no se había situado en el conjunto de los países que precozmente concedieron el sufragio (Ecuador, Cuba, Brasil y Uruguay), a mediados del siglo pasado ostentaba un número de representantes que no era igualado en la cuenca regional, ni en la enorme mayoría de los países europeos.

Cabe recordar que la Unión Cívica Radical, el principal partido contendiente con el peronismo, no incluyó ninguna mujer en las listas de candidaturas nacionales, tal vez como una manera de mostrar su completa desaprobación al régimen. Por su parte, la Rama Femenina era una de las más vigorosas y actuantes, un instrumento fundamental en manos de Evita, que a la sazón se había inscrito en las mayorías populares de manera casi sacramental. Hubo una suerte de feminización de la política como nunca había ocurrido antes, aunque no significó, en absoluto, la feminización del poder. Evita hacía ostensible la incontestable tradición de la politización de las mujeres en la sociedad argentina. Las devociones reverenciales que se le dedicaron a Evita, constituyeron una marca central del escenario político y social de la Argentina de mediados del siglo pasado. Su muerte, en julio de 1952, fue un mazazo para las sensibilidades populares, pero también para la forja de sentidos más audaces por parte de su gran creación, la Rama Femenina.

\section{Coda}

El feminismo argentino surgió de modo temprano, a fines del XIX, con la incorporación de mujeres que en gran mayoría pertenecían al socialismo o a manifestaciones del librepensamiento, y cundió especialmente entre las más letradas. En la agenda de los derechos figuró la cuestión del sufragio, aunque los acuerdos sobre su extensión demoraron algún tiempo ya que una parte de las feministas abogaba por el voto universal en iguales condiciones que los varones, pero otra, tal vez mayoritaria, afirmaba que debía experimentarse a través de etapas sucesivas. La condición de la ilustración de las mujeres parecía ser decisiva en este segundo grupo. Pero después de la Gran Guerra, la enorme mayoría de las adherentes sostuvo que la ciudadanía debía conquistarse en igualdad con los varones, asistiéndose a un empinado movimiento pro sufragio que alcanzó inclusive a sectores de mujeres más conservadores.

Es que en el transcurso de la época, a pesar de los espíritus medrosos que sostenían que las mujeres votarían según la opinión más retardataria, pareció abrirse un cauce mayor para la recepción de los reclamos feministas. El voto fue debatido por la Cámara Baja en 1932 de modo exitoso, pero el Senado no trató la cuestión. Durante el interregno 1933-1944 las feministas argentinas, en su gran mayoría, cambiaron el eje de las movilizaciones debido a que la preocupación central fue la lucha antifascista, la ayuda a los refugiados de la Guerra

24 Civil Española y la acción para impedir el ascenso de las derechas en el país. 
En la coyuntura surgió el peronismo -visto como una forma del fascismo por las fuerzas liberales y de izquierda- y su propuesta de voto femenino fue azuzada en buena medida por la propia Eva Perón, figura central de la escena del nuevo régimen. Se arribó así, a la sanción del sufragio en septiembre de 1947, la primera vez que las mujeres se expresaron en las urnas en 1951, gracias a la acción desplegada por la Rama Femenina del peronismo, pudiendo ocupar una significativa proporción de las bancas del Congreso, circunstancia que colocó al país en una situación singular y no solo en América Latina. Pero las feministas, opositoras al régimen, no figuraron en esa leva de las primeras legisladoras.

\section{Referencias}

Barrancos, Dora. (1989). Anarquismo, educación y costumbres en la Argentina de principios de siglo. Buenos Aires: Contrapunto.

Barrancos, Dora. (1996). La escena iluminada. Ciencias para trabajadores 1890-1930. Buenos Aires: Plus Ultra.

Barrancos, Dora. (2002). Inclusión/Exclusión. Buenos Aires: Fondo de Cultura Económica.

Barrancos, Dora. (2005a). Socialismo y sufragio femenino. Notas para su historia (1890-1943). En Carlos Herrera y Hernán Camarero. (Eds). El Partido Socialista en Argentina. Sociedad, política e ideas a través de un siglo. Buenos Aires: Prometeo.

Barrancos, Dora. (2005b). Primera recepción del término "feminismo" en la Argentina. Labrys, revista de estudos feministas/études féministes, No. 8. Dossier dirigido por María Luisa Femenías. Recuperado el 18 de febrero del 2014 de: http://www.tanianavarroswain.com.br/labrys/labrys8/princi$\mathrm{pal} / \mathrm{dora} \cdot \mathrm{htm}$

Barrancos, Dora. (2007). Mujeres en la sociedad argentina. Una historia de cinco siglos. Buenos Aires: Sudamericana.

Barrancos, Dora. (2008). Mujeres, entre la casa y la plaza. Buenos Aires: Sudamericana.

Barry, Carolina. (2009). Evita capitana. El partido peronista femenino, 19491955. Buenos Aires: Eduntref.

Bellota, Aracelli. (2000). Julieta Lanteri, la pasión de una mujer. Buenos Aires: Planeta.

Dujovne Ortiz, Alicia. (1995). Eva Perón: la biografía. Buenos Aires: Aguilar. 
Lavrin, Asunción. (2005). Mujeres, feminismo y cambio social en Argentina, Chile y Uruguay 1890-1940. Santiago de Chile: Centro de Investigaciones Diego Barros Arana.

Mc Gee Deutsch, Sandra. (2007). Desafiando al antisemitismo y a la derecha: la participación de las mujeres judías en grupos antifascistas en Argentina, 1935-1945. Ponencia presentada en la Latin American Jewish Studies Association, Buenos Aires, 29 al 31 de julio.

Navarro, Marysa. (1981). Evita. Buenos Aires: Corregidor.

Palermo, Silvana. (1998). El sufragio femenino en el congreso nacional: ideologías de género y ciudadanía en la Argentina (1916-1955). Boletín del Instituto de Historia Argentina y Americana Dr. Emilio Ravignani, 151-178.

Perrot, Michele. (2008). Mi historia de las mujeres. Buenos Aires: Fondo de Cultura Económica.

Pichel, Eva. (1993). Evita íntima. Buenos Aires: Planeta.

Valobra, Adriana. (2010). Del hogar a las urnas. Recorridos de la ciudadanía política argentina, 1946-1955. Rosario: Prohistoria.

Vallejo, Gustavo. (2007). Escenarios de la cultura científica argentina. Ciudad y Universidad (182-1955). Madrid: Consejo Superior de Investigaciones Científicas. 